\title{
Vascular malforma- tions part 1 - normal and abnormal vascular development
}

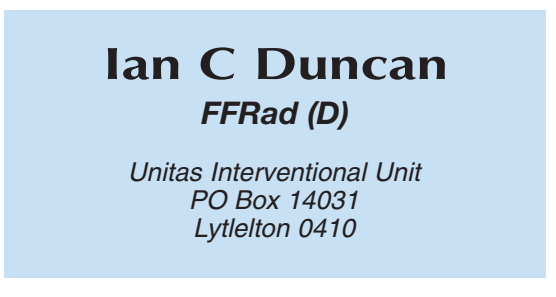

\section{Introduction}

The existence of all multicellular organisms on our planet is in part attributable to the development of a circulatory system. In vertebrates this has evolved to a highly complex structure whose function is to supply oxygen and nutrients to every living cell within the body, making it the largest single 'organ' within our bodies. Its development and growth are stimulated by a combination of genetic and environmentally controlled factors. The vasculature also undergoes a constant process of maintenance and repair throughout life, with the entire vascular tree being replenished on average every 3 years. This article reviews some of the basic biology of normal and abnormal vascular development and how this relates to the development of vascular anomalies.

\section{Vasculogenesis and angiogenesis}

Vasculogenesis is the first stage of development of the vascular tree. This involves the differentiation of one or more mesodermally-derived vascular precursor cell types into angioblasts, the migratory precursors of endothelial and vascular smooth muscle cells. ${ }^{1-3}$ Differentiation of angioblasts is followed by the association and assembly of endothelial cells to form the primitive vascular plexus.

Angiogenesis is the formation of new vessels by sprouting or splitting of pre-existing vessels. Sprouting (or budding) angiogenesis occurs in various phases. During the activation phase there is disassembly of the vessel wall followed by proliferation and migration of endothelial cells into the surrounding extracellular matrix. During the resolution, or stabilisation, phase, the proliferation and migration of endothelial cells is halted and the vessel walls, both new and old, are then re-assembled and stabilised. Failure to stabilise the new immature vessel leads to it undergoing apoptosis (cell death). Non-sprouting angiogenesis (also termed splitting angiogenesis or intussusception) is characterised by the growth of interstitial cellular columns into the lumen of an already existing vessel so as to partition or split the vessel and thus remodel the local vascular network.

Angiogenesis is dependent upon the complex interaction between endothelial and smooth muscle cells and various growth (angiogenic) fac- tors (Table 1) and the extracellular matrix (ECM). The ECM consists of a complex three-dimensional network of (structural) proteins, polysaccharides and signal molecules surrounding the endothelial cell layer and contained by the basal lamina. During the activation stage of angiogenesis the ECM 'softens' and ruptures allowing endothelial cells to migrate and proliferate. During and after the resolution stage the ECM helps to reconstitute the vessel wall and thereafter to maintain its stability. ECM factors are listed in Table II.

Angiogenesis during the embryonic period is driven by the growing metabolic demand within the embryo due to the increase in organ size as well as the increase in distance between capillaries and cells resulting in reduced oxygen diffusion in the developing tissues. After birth angiogenesis is driven by normal physiological events such as growth, exercise, the menstrual cycle or pregnancy, or by pathological conditions such as tumour, ischaemia, inflammation, metabolic derangements, trauma, etc.

Arteriogenesis is the remodelling of pre-existing arteriolar collateral networks with coalescence of these to form larger conductance arteries which serve to redirect blood flow toward areas of higher metabolic demand.

Vasculogenesis, angiogenesis and arteriogenesis thus form a continuum involved not only in the primary development but also the maintenance and repair of blood vessels throughout life. The regulation of these processes is brought about by a genetic and environmentally determined balance between a multitude of proangiogenic and antiangiogenic factors and the extracellular matrix 


\section{Table I. Angiogenetic growth factors}

1. Vascular endothelial growth factor (VEGF- A-E and PIGF)

- Stimulates angiogenesis

- Stimulates endothelial protease production

- Causes controlled microvascular permeability to plasma proteins

- Stimulates the formation of angioblasts from precursor cells

- $\quad$ VEGF-C and-D are associated with lymphangiogenesis

2. Angiopoietins: (Ang 1 to 4 )

- $\quad$ Are ligands for the endothelium-specific receptors TIE2

- $\quad$ Stimulate vascular remodelling (Ang1 + TIE2)

- $\quad$ Stimulate endothelial maturation and stabilisation (Ang1 + TIE2 with VEGF)

- Ang2 with VEGF produces loss of adhesion of endothelial cells allowing their migration to occur

3. Fibroblast growth factor : (aFGF, bFGF)

- Are potent stimulators of endothelial cell migration, proliferation, sprouting and tube formation

- $\quad$ Are mainly involved with vascular maintenance and repair

4. Platelet derived factor (PDGF)

- Promotes proliferation and migration of endothelial cells, vascular smooth muscle cells and pericytes

5. Transforming growth factor: (TGF $\alpha$ and $\beta$ )

- $\quad$ TGF $\beta$ is a potent inhibitor of endothelial cell migration and proliferation

6. Others, including EGF, TNF $\alpha$, ephrins, etc.

\section{Table II. Extracellular matrix factors ${ }^{2}$}

1. Coagulation and fibrinolytic factors:

- Regulation of coagulation is required to avoid thrombosis or haemorrhage during neoangiogenesis

2. Metalloproteinosis (MMP)

- Are proteolytic enzymes that cause degredation of the basal lamina and extracellular matrix before endothelial cell migration

3. Integrins:

- $\quad$ Promote cell-cell and cell-matrix adhesion

(ECM). A full description of these growth factors and the ECM (structural proteins) and their role in vasculogenesis/angiogenesis is beyond the scope of this review, but for further information the reader is referred to the excellent reviews on this subject by Kubis and Levy ${ }^{1,2}$ and Harrigan. ${ }^{3}$

The differentiation of primitive vessels into arteries, veins or capillaries is determined by flow patterns and is regulated by a class of molecules known as the ephrins. The development of congenital arteriovenous malformations may thus be related to defects in the ephrin signalling system. Local environmental stimuli including shear stress or ischaemia in arterio-venous malformations (AVMs) may stimulate angiogenic factors and produce abnormal vascular remodelling. Differences in the expression of various structural proteins and angiogenic factors are seen in different cerebral vascular malformations., ${ }^{3,4}$ For instance the endothelial and subendothelial layers of AVMs express more laminin and collagen IV than those in low-flow cavernous malformations, whereas cavernous malformations express more fibronectin than AVMs. Fibronectin and laminin are structural proteins found in the basement membranes of normal vessels, and are thought to maintain the structural integrity of vessel walls by anchoring endothelial cells to the underlying internal elastic membrane and smooth muscle layers. Fibronectin tends to predominate in the initial stages of angiogenesis (activation stage) where immature vessels with non-adherent proliferating endothelial cells exist in a fibronectin -rich extracellular matrix. Laminin is found mainly during the maturation phase of angiogenesis where more mature vessels with adherent nonproliferating endothelium are found. Therefore it can be concluded that cavernous malformations are angiogenically more immature than AVMs. This relative immaturity of the walls of a cavernous malformation results in a high degree of fragility and thus a tendency to bleed in the absence of increased haemodynamic forces. ${ }^{4}$ In addition to the differences in the levels of various structural proteins between the two malformation types there is also a difference in the expression of angiogenic factors. Although vascular endothelial growth factor (VEGF), b fibroblastic growth factor (bFGF) and transforming growth factor $\alpha$ (TGF $\alpha$ ) are expressed in both AVMs and cavernous malformations in keeping with the active angiogenic processes associated with their development, 
bFGF is expressed in the endothelia of CMs but not in the endothelia of AVMs. Glial cells adjacent to AVMs express VEGF whereas those adjacent to CMs express bFGF and TGF $\alpha$ but do not express VEGF. Venous angiomas, by comparison, do not express growth factors but do express structural proteins of the mature phase of angiogenesis. ${ }^{4}$

The above examples show distinct biological differences between the different malformation types related to the variable expression of structural matrix proteins and angiogenic factors within each. It would be an oversimplification to claim that a deficiency or functional abnormality of one or more specific structural proteins or angiogenic factors will lead to the development of a specific malformation type as the aetiological development of vascular malformations is undoubtedly multifactorial, depending upon a number of factors including genetic defects, environmental effects (biological and mechanical) and trigger agents or events. ${ }^{5}$

\section{Genetic factors in vascular malformations}

There is primarily a basic underlying genetic control of vasculogenic and angiogenic processes, modified by metabolic demand and other physiological influences. A clear link between the development of vascular malformations and an identifiable genetic defect has been shown to date in several distinct conditions. ${ }^{6,7}$

1. Hereditary haemorrhagic telangiectasia (HHT) (Osler-WeberRendu): This is a multisystemic angiodysplasia which is inherited as an autosomal dominant trait with varying degrees of penetrance and expressivity. Defects in two genes have thus far been identified as being responsible for the induction of the vascular malformations seen in HHT. ${ }^{8}$ These genes are endoglin (chromosome 9, HHT 1) and ALK-1 (chromosome 12, HHT 2), both of which encode for vascular endothelial transmembrane receptors of transforming growth factor $-\beta$ (TGF- $\beta$ ). ${ }^{1}$ TGF- $\beta$, in turn, plays a role in endothelial cell resolution via activation of the ALK-1 pathway, and endothelial cell activation via activation of the ALK-5 pathway. A reduction of the levels of endoglin in HHT-1 may lead to a decrease in TGF- $\beta$ levels, affecting both the ALK-1 and ALK-5 pathways. The ALK-5 pathway, however, has a higher sensitivity to the remaining TGF- $\beta$ than the ALK-1 pathway thereby preferentially stimulating endothelial cell activation. In HHT-2 reduced ALK-1 proteins lead to preferential relative overactivity of the ALK-5 pathway again stimulating endothelial cell activation. This activation then stimulates the production of the angiogenic inducer vascular endothelial growth factor (VEGF). Levels of VEGF have been shown to be significantly elevated in patients with HHT. ${ }^{9}$ Either interruption of TGF- $\beta$ function or increased levels of VEGF or other related angiogenic factors may thus play a role in the development and growth of the various vascular malformation types seen in HHT.

2. Venous malformations: Venous malformations result from mutations in at least 2 genes on chromosomes 1(1p21-22) and 9 (9p21-22/TIE2). The latter gene, TIE2, encodes an endothelial cell surface receptor for a group of extracellular signalling mol- ecules called the angiopoeitins which regulate the maturation of primitive vessels. In a number of patients with venous malformations the disease is inherited as an autosomal dominant trait. The Blue Rubber Bleb Nevus syndrome (Bean syndrome) is an inherited disorder where patients develop venous malformations in the skin, gastrointestinal tract, brain and other organs.

3. Arterial malformations in neurofibromatosis type I: NF I results from mutations on chromosome 17. The affected gene, neurofibromin, encodes an intracellular signalling protein that has a role in embryonic vascular development.

\section{Familial cavernous malforma-} tion: Cavernous malformations (CMs) can be classified as either sporadic or familial. Some $20-30 \%$ of patients with CMs in North America may have the familial form in which an autosomal dominant pattern of inheritance is seen. The genetic mutation responsible for the development of these CMs has been identified on the long arm of the $7 \mathrm{q}$ chromosome. ${ }^{10}$ Further point mutations have been identified including two on the short arm of chromasome 7(7p15-13) and the long arm of chromosome $3(3 \mathrm{q}$ 25.2.27)(CCM52+3). ${ }^{11,12}$ The first of these genes, CCM1, has been identified more recently as encoding the KRIT1 protein which plays a role in intercellular signalling particularly to endothelial cells. ${ }^{13}$

Thus the importance of the above diseases lies in the fact that distinct genetic mutations have now been identified that have led directly to the phenotypic expression of vascular malformation development. All of the above are inherited as autosomal dominant traits, rendering them easy 
to identify as genetic abnormalities. Others having an autosomal recessive or polygenic pattern of inheritance will undoubtedly be more difficult to identify, but it is probable that as time progresses further specific genetic defects related to the development of vascular malformations will be discovered. ${ }^{6,7}$

\section{Lymphangiogenesis}

Lymphatic vessels develop parallel to blood vessels and drain tissue fluid that results from normal leakage from blood vessels. They arise by both sprouting from existing embryonic veins as well as by the in situ differentiation of lymphatic endothelial cells from lymphangioblasts. ${ }^{14}$ Certain specific proteins have been shown to mediate the development and growth of lymphatic endothelium. These include vascular endothelial growth factors $\mathrm{C}$ and D (VEGF-C and VEGFD) and the receptor VEGFR-3(or FLT-4).${ }^{15}$ It is thought that VEGF-C induces lymphatic growth mediated by VEGFR-3. Other genes that have been shown to be relatively specifically expressed in lymphatic endothelial cells include Prox 1, Podoplanin and LYVE-1. Approximately 35\% of patients with primary lymphoedema have a positive family history for the disease. Primary congenital lymphoedema (Milroy's disease) results from mutations on the VEGFR-3 (or FLT-4) gene found on chromosome 5(5q35.3). Abnormal VEGFR-3 activity leads to reduced binding of ligands such as angiopoeitin-1(Ang-1). The angiopoeitins are ligands for TIE-2, an endothelial cell receptor important in the stabilisation and maturation of the vascular network in embryos. Ang-1 also seems to inhibit VEGFinduced vessel permeability. This would thus lead to increased lymphatic permeability and the development of lymphoedema. Late onset lymphoedema (lymphoedema praecox, Meige's disease) develops at the onset of puberty. This syndrome together with others including lymphoedema distichiasis (lymphoedema with abnormal hairs from eyelid meibomian glands), lymphoedema and ptosis, and yellow nail syndrome are all linked to inactivation mutations of the FOXC2 (MFH-1) gene on chromosome 16 (16q24).

Lymphatic malformations, in contrast to lymphoedemas, are true malformations composed of dilated lymphatic channels or vesicles filled with fluid but which do not connect with lymphatic vessels. They are present at birth and typically enlarge in the presence of infection. ${ }^{14}$ They demonstrate a normal endothelial cell replication cycle unlike lymphangiomas which are hypercellular tumours of lymphatic origin. ${ }^{16}$ Because of the developmental association between veins and lymphatics, mixed venolymphatic malformations may occur.

\section{Endothelial proliferation}

Endothelial cell proliferation occurs as part of the normal angiogenic or lymphangiogenic process. The proliferation of endothelial cells is induced during the activation phase of angiogenesis by factors such as platelet derived growth factor (PDGF) and is inhibited during the resolution phase by factors such as transforming growth factor $\beta 1$ (TGF $\beta 1) .{ }^{1}$ Mulliken and Glowacki ${ }^{16}$ classified paediatric vascular malformations based on endothelial cell characteristics, specifically the rate of endothelial cell turnover. They differentiated between haemangiomas that are characterised by endothelial cell hyperactivity during the proliferative phase (rapid neonatal growth) followed by diminishing cellularity during the involuting phase later in life, and vascular malformations that are characterised by a normal rate of endothelial cell turnover (compared with normal blood vessel endothelial cell turnover). Thus the malformations remain true malformations lined with mature endothelial cells, whereas the haemangiomas are essentially endothelial cell tumours. The lymphatic equivalents are lymphangioma and lymphatic malformations, again differentiated according to lymphatic endothelial cell growth characteristics. The growth and development of an infantile haemangioma mimics the growth of the normal vascular tree in that early lesions are highly cellular with plump endothelial cells lining vascular spaces with very small lumina, and that as they mature the endothelium becomes flattened and the vascular spaces enlarge. ${ }^{17}$ Thus haemangiomas represent a form of 'runaway' neoplastic endothelial cell proliferation. Increased endothelial proliferation is, however, also seen to occur to a much lesser degree in vascular malformations as well. The rate of endothelial cell turnover is seven times greater in cerebral AVMs than in normal cerebral blood vessels. ${ }^{18}$ Increased endothelial cell turnover has also been demonstrated in cerebral cavernous malformations. ${ }^{19}$ Thus although increased, endothelial cell growth rates are nowhere near the scale of those seen in haemangiomas, remaining the key differentiating feature between the two vascular abnormalities (Table III). 
Table III. Characteristics of haemangiomas and vascular malformations

\begin{tabular}{lcc}
\hline & Angiogenesis & $\begin{array}{l}\text { Endothelial cell } \\
\text { proliferation }\end{array}$ \\
\hline Haemangiomas & + & ++++ \\
Vascular malformations & + & + \\
\hline
\end{tabular}

\section{The future}

There is currently a tremendous amount of research being conducted into the role of angiogenesis and genetic mutations in the development of vascular malformations, tumours, inflammatory diseases and other pathological processes. Presently under investigation are a number of anti-angiogenic agents being tried for the treatment of various tumours. ${ }^{3}$ Additionally pro-angiogenic agents may someday play an important role in combating the effects of tissue or organ ischaemia. Future treatments for vascular and lymphatic malformations will undoubtedly also involve gene-based therapies. ${ }^{?}$

\section{References}

1. Kubis N, Levy BI. Vasculogenesis and angiogenesis: molecular and cellular controls. Part 1: Growth factors. Interventional Neuroradiology 2003; 9: 227-237.

2. Kubis N, Levy BI. Vasculogenesis and angiogenesis: molecular and cellular controls. Part 2: Interactions between cell and extracellular environment. Interventional Neuroradiology 2003; 9: 239-248.

3. Harrigan MR. Angiogenic factors in the central nervous system. Neurosurgery 2003; 53: 639-661.

4. Kilic T, Pamir MN, Kullu S, Eren F, Ozek MM, Black PM. Expression of structural proteins and angiogenic factors in cerebrovascular anomalies. Neurosurgery 2000; 46: 1179-1191.

5. Lasjaunias PL. Segmental identity and vulnerability in cerebral arteries. Interventional Neuroradiology 2000; 6: 113-124.

6. Shovlin CL. Genetic aspects of cerebrovascular diseases. Interventional Neuroradiology 2000; 6: 107-111.

7. Vikkula M, Boon LM, Mulliken JB. Molecular genetics of vascular malformations. Matrix Biol 2001; 20: 327-335.

8. Begbie ME, Wallace GMF, Shovlin CL. Hereditary haemorrhagic telangiectasia (OslerWeber-Rendu syndrome): a view from the 21st century. Postgrad Med J 2003; 79: 18-24.
9. Cirulli A, Liso A, D'Ovido F, et al. Vascular endothelial growth factor serum levels are elevated in patients with hereditary haemorrhagic telangiectasia. Acta Haematol 2003; 110: 29-32.

10. Dubovsky J, Zabramski JM, Kurth J, et al. A gene responsible for cavernous malformations of the brain maps to chromosome 7q. Hum Mol Genet 1995; 4: 453-458.

11. Craig HD, Günel M, Cepeda O, et al. Multilocus linkage identifies two new loci for a Mendelian form of a stroke, cerebral cavernous malformation, at 7p15-13 and 3q25.2-27. Hum Mol Genet 1998; 12: 1851-1858.

12. Günel M, Awad IA, Finberg K, et al. Genetic heterogeneity of inherited cerebral cavernous malformation. Neurosurgery 1996; 38: 1265-1271.

13. Couteulx SL, Jung $\mathrm{H}$, Labauge $\mathrm{P}$, et al. Truncating mutations in CCM 1 , encoding KRIT 1, cause hereditary cavernous angiomas. Nat Genet 1999; 23: 189-193.

14. Brouillard P, Vikkula M. Vascular malformations: localized defects in vascular morphogenesis. Clin Genet 2003; 63: 340-351.

15. Karkkainen MJ, Jussila L, Ferrell RE, Finegold DN, Alitalo K. Molecular regulation of lymphangiogenesis and targets for tissue oedema. Trends in Molecular Medicine 2001; 7: 18-22.

16. Mulliken JB, Glowacki J. Hemangiomas and vascular malformations in Infants and children: A classification based on endothelial characteristics. Plast Reconstr Surg 1982; 69: 412-419.

17. Requena L, Sangueza OP. Cutaneous vascular proliferations. Part II. Hyperplasia and benign neoplasms. J Am Acad Dermatol 1997; 37: 887919.

18. Hashimoto T, Mesa-Tejada R, Quick CM, et al. Evidence of increased endothelial cell turnover in brain arteriovenous malformations. Neurosurgery 2001; 49: 124-132.

19. Sure U, Butz N, Schlegel J, et al. Endothelial proliferation, neoangiogenesis and potential de novo generation of cerebrovascular malformations. J Neurosurg 2001; 94: 972-977. 